\section{Cobertura do Sistema de Informações sobre Nascidos Vivos e potenciais fontes de informação em municípios de pequeno porte em Minas Gerais, Brasil}

\section{The coverage of the Live Births Information System and potential sources of information in small-scale municipalities in the Brazilian State of Minas Gerais}

Anne Marielle Girodo1

Deise Campos 2

Sonia Duarte de Azevedo Bittencourt 3

Célia Landmann Szwarcwald 4

Elisabeth Barboza França 5

${ }^{1}$ Secretaria Municipal de Saúde de Belo Horizonte. Av. Afonso Pena, 2336. $9^{\circ}$ andar. Funcionários. Belo Horizonte, MG, Brasil.

E-mail: annemarielle@gmail.com

2 Gerência de Ensino e Pesquisa da Fundação Hospitalar do Estado de Minas Gerais. Grupo de Pesquisas em Epidemiologia e Avaliação em Saúde. Faculdade de Medicina. Universidade Federal de Minas Gerais. Belo Horizonte, MG, Brasil.

3 Departamento de Epidemiologia e Métodos Quantitativos em Saúde. Escola Nacional de Saúde Pública. Fundação Oswaldo Cruz. Rio de Janeiro, RJ, Brasil.

4 Instituto de Comunicação e Informação Científica e Tecnológica em Saúde. Fundação Oswaldo Cruz. Rio de Janeiro, RJ, Brasil.

5 Programa de Pós-graduação em Medicina Preventiva e Social Faculdade de Medicina. Universidade Federal de Minas Gerais. Belo Horizonte, MG, Brasil.

\section{Resumo}

Objetivos: estimar a cobertura do Sistema de Informações sobre Nascidos Vivos (SINASC) e a contribuição de potenciais fontes de informação de amostra de municípios da mesorregião do Jequitinhonha, Minas Gerais, em 2008.

Métodos: a lista de nascidos vivos (NV) informados ao SINASC foi complementada por NV localizados por busca ativa em cartórios, unidades de saúde, secretarias de assistência social, informanteschave das comunidades, parteiras tradicionais, igrejas, farmácias e arquivos das secretarias municipais de saúde. A cobertura foi calculada a partir da relação de NV informados ao SINASC e total de NV após busca ativa.

Resultados: os resultados indicaram uma cobertura precária do SINASC, sendo inferior a $60 \% \mathrm{em}$ três dos cinco municipios estudados. Quase um quarto dos $N V$ subenumerados não teve Declaração de Nascido Vivo (DN) emitida. As principais fontes de informação foram os cartórios, hospitais e Unidades Básicas de Saúde (UBS), correspondendo juntas ao total de $60 \%$ dos NV localizados pela busca ativa.

Conclusões: o produto da busca ativa de NV indicou problemas na qualidade da informação dos registros vitais em municípios de pequeno porte, relacionados principalmente a problemas na coleta e no fluxo das informações do SINASC nesses municipios. Palavras-chave Avaliação de serviços de saúde, Nascidos vivos, Sistemas de informação, Sub-registro 


\section{Introdução}

Estatísticas vitais são indispensáveis instrumentos na elaboração de indicadores de saúde e demográficos, permitindo subsidiar o planejamento, execução e avaliação das ações de saúde.1,2 Indicadores como o coeficiente de mortalidade infantil e a razão de mortalidade materna, a proporção de baixo peso ao nascer e as taxas de natalidade e de fecundidade, amplamente avaliados e discutidos nas políticas públicas, utilizam a informação de nascidos vivos, que por sua vez deve ser de qualidade e oportuna. ${ }^{3-5}$ Considerando a importância desses registros, a partir de 1974 o Brasil passou a ter informação de nascimentos através do registro civil, com coleta e consolidação dos dados de responsabilidade do Instituto Brasileiro de Geografia e Estatística (IBGE).

Em 1990, o Ministério da Saúde implantou o Sistema de Informações sobre Nascidos Vivos (SINASC), investindo em um sistema que permitisse não somente obter o número de nascidos no território nacional, mas também que subsidiasse informações que possibilitem a execução de ações relacionadas à saúde da mulher e da criança, em todos os níveis do Sistema Único de Saúde (SUS). 6 A partir do SINASC é possível conhecer o perfil epidemiológico dos nascimentos, tanto dos municípios quanto do país, através de variáveis como tipo de parto, duração da gestação, peso ao nascer, idade e escolaridade da mãe e local do parto.

Estudo divulgado pelo IBGE mostrou que, sob o ponto de vista de qualidade, o SINASC tem apresentado melhorias a cada ano, atingindo cobertura de 92\% em 2006 no Brasil como um todo. 5 Apesar dos avanços, o SINASC ainda não contempla todos os registros de nascimentos no país, 7,8 havendo uma desigualdade na sua distribuição espacial, sendo particularmente maior nas Regiões Norte e Nordeste. $4,8-10$

Historicamente, tendo em vista as limitações relativas à subenumeração de nascidos vivos $(\mathrm{NV})$, estimativas indiretas do número de $\mathrm{NV}$ eram muito utilizadas no cálculo de indicadores demográficos e de saúde. Estas estimativas eram baseadas em informações de censo e de inquéritos como as Pesquisas Nacionais por Amostra de Domicílio (PNAD), ambos realizados pelo Instituto Brasileiro de Geografia e Estatística.2,10,11 Entretanto, a qualidade destas estatísticas é dependente do tamanho das amostras populacionais e encontram limitações em sua aplicação quando se trata de municípios menores. Ou seja, não fornecem informações desagregadas para essas unidades territoriais, que são os locais onde se concentram os maiores problemas de saúde materno-infantil. 4

Em 2000, a Organização das Nações Unidas (ONU) propôs, dentre os Objetivos de Desenvolvimento do Milênio (ODM), a redução da mortalidade infantil e materna. Tendo assumido o compromisso do cumprimento dos ODM junto à ONU, o Brasil desencadeou programas buscando intensificar ações na esfera da organização dos serviços de saúde. ${ }^{12,13}$ Entretanto, uma das dificuldades para a avaliação precisa da magnitude da mortalidade infantil e materna, cujos indicadores calculados pelo método direto utilizam a informação de nascidos vivos e de óbitos infantis registrados no SINASC e no Sistema de Informações sobre Mortalidade (SIM), é exatamente a disponibilidade de dados com grau satisfatório de confiabilidade para estimar e analisar a evolução dos indicadores nas áreas mais carentes.

Diante dessas questões, as estimativas de nascimentos e óbitos pelo método direto têm importância crescente e fomentam o interesse pelo uso de informações vitais de registro contínuo.4,8,9 Por conseguinte, torna-se indispensável e prioritária a realização de investigações para dimensionar a cobertura desses eventos, bem como conhecer pontos críticos e necessidades para o adequado funcionamento dos sistemas de informação em saúde.

É nesse contexto que o Ministério da Saúde vem propondo iniciativas para a avaliação e melhoria da cobertura e da completude das informações sobre óbitos e nascimentos no país.14 Estudos que propõem o relacionamento de banco de dados, o método de captura e recaptura de registros e a busca ativa de eventos são algumas dessas iniciativas que vêm mostrando resultados de melhoria na cobertura das regiões onde eles foram aplicados.4,8,14-16 Dentre esses, o método da busca ativa de nascimentos não informados ao Ministério da Saúde tem a vantagem de além de permitir localizar esses eventos possibilita ainda complementar dados para o preenchimento de uma Declaração de Nascido Vivo (DN).

Em Minas Gerais, o Vale do Jequitinhonha é uma região amplamente conhecida pelos seus baixos indicadores sociais, que a colocam entre as mais carentes do país. ${ }^{17}$ Em 2001, estudo realizado a partir do Sistema de Informações Hospitalares (SIH-SUS) indicou ser essa área afetada por problemas de subenumeração e de baixa confiabilidade das informações dos eventos de saúde. ${ }^{15}$ Dessa forma, a realização do presente estudo tem como objetivo estimar a cobertura de NV no SINASC pelo método de busca ativa de nascimentos em municípios de 
pequeno e médio porte do Vale do Jequitinhonha, e identificar as principais fontes de informação para registros desse evento.

\section{Métodos}

O presente trabalho é parte integrante da pesquisa "Condições de nascimento, de assistência ao parto e aos menores de um ano em uma amostra de municípios de médio e pequeno porte nas Regiões Norte, Nordeste do Brasil e no Vale do Jequitinhonha em Minas Gerais" da Fundação Oswaldo Cruz.

Na primeira etapa do estudo, todos os municípios da Mesorregião Vale do Jequitinhonha, Minas Gerais, foram classificados por adequação das informações como "satisfatório", "não satisfatório" e "deficiente", segundo método desenvolvido por Andrade e Szwarcwald, ${ }^{9}$ para o triênio 2005-2007. Para tanto, foram utilizados quatro indicadores 2 : coeficiente de mortalidade geral padronizado por idade; desvio médio relativo do coeficiente geral de mortalidade; razão entre nascidos vivos informados e estimados e desvio médio relativo da taxa de natalidade. Na segunda etapa, foi selecionada uma amostra probabilística de municípios, estratificada por adequação das informações.

Os municípios selecionados para o estudo foram: Araçuaí (37.262 habitantes), Felício dos Santos (5.857 habitantes), Joaíma (15.391 habitantes), Jordânia (11.209 habitantes) e Novo Cruzeiro (31.264 habitantes). 18 Segundo o Censo Demográfico de 201018 os municípios estudados têm de $55 \%$ a $68 \%$ da população residente com renda declarada inferior a meio salário mínimo; taxas de analfabetismo próximas de $30 \%$ em dois municípios; cobertura mediana de esgotamento sanitário de apenas $35,6 \%$; cobertura de abastecimento de água de $69 \%$ e índice de desenvolvimento humano (IDH) alto em somente dois municípios dos cinco selecionados.

Uma das etapas iniciais do trabalho de campo foi a elaboração de uma lista nominal de nascidos vivos em 2008 de residentes dos municípios selecionados, fornecida pela Secretaria de Vigilância em Saúde do Ministério da Saúde (SVS-MS). A lista nominal foi elaborada a partir dos nascimentos informados ao SINASC e Sistema de Internações Hospitalares (SIH). Os dados coletados no processo de busca ativa foram utilizados para completar as listas nominais originais de nascidos vivos. ${ }^{8}$

A coleta de dados envolveu todos os NV no período de $1^{\circ}$ de janeiro e 31 de dezembro de 2008, filhos de mães residentes dos municípios da amostra. Os dados foram coletados localmente nos meses de dezembro de 2010 e janeiro de 2011, através de um processo de busca ativa. Buscaram-se tanto as DN emitidas e não inseridas no SINASC, como a ocorrência de nascimentos vivos que não geraram as respectivas DN. Os registros foram utilizados para completar as listas nominais originais de nascidos vivos.

Para a coleta, foram investigadas todas as possíveis fontes de informações utilizando instrumentos padronizados, sendo elas: cartórios; igrejas (registros de batismo e pastoral da criança); Unidades Básicas de Saúde - UBS (prontuários, cartão espelho de vacinação, teste do pezinho), hospitais (livros de estatísticas hospitalares, livros de controle de admissão e alta, livro da sala de parto, guias de Autorização de Internação Hospitalar - AIH e, prontuários); agentes comunitários de saúde, profissionais do PSF (informações registradas no Sistema de Informação da Atenção Básica - SIAB e informações verbais); informantes chaves como parteiras tradicionais, líderes comunitários, curandeiros, rezadeiras; serviços municipais de assistência social (Bolsa Família, auxílio enxoval, auxílio leite); além de farmácias. Também foram investigados cartórios e hospitais de municípios vizinhos onde poderiam ter ocorrido nascimentos daquele município.

Os NV localizados através da busca ativa foram pareados com a lista nominal disponibilizada pela SVS/MS e realizada a confirmação dos eventos através de pesquisa do registro em cartório ou em unidade básica de saúde. Os nascimentos encontrados sem registro de ocorrência no SINASC e/ou SIH-SUS, cartórios ou unidade de saúde foram investigados por meio de visita aos domicílios para sua confirmação, mediante entrevista.

As notificações de NV obtidas por busca ativa foram pareadas com as DN disponíveis na base de dados do SINASC, utilizando como variáveis-chave a data de nascimento da criança, nome da mãe e endereço. Para o cálculo da cobertura, utilizou-se a razão entre os nascidos vivos presentes no SINASC e o total acrescido do número de $\mathrm{NV}$ identificado na pesquisa.

Cobertura $=$ (número de $\mathrm{NV}$ informado ao SINASC) / (número de NV informado ao SINASC + número de NV identificado na busca ativa) * 100\%

O projeto foi aprovado pelo Comitê de Ética em Pesquisa em Pesquisa do Centro de Pesquisa René Rachou da Fundação Oswaldo Cruz, conforme a Carta de Aprovação de nº 26/2009 - CEP/CPQRR. 


\section{Resultados}

Foram localizados na busca ativa $485 \mathrm{NV}$ que não haviam sido informados ao SINASC, correspondendo a quase um quarto $(24,5 \%)$ do total de 1980 NV em 2008 de mães residentes nos cinco municípios da pesquisa. Essa subenumeração foi de 7,9\% no município de Araçuaí, a 70,2\% no município de Jordânia, resultando em coberturas muito baixas, como as encontradas nos municípios de Felício dos Santos, Joaíma e Jordânia $(58,3 \%, 59,2 \%$ e $29,8 \%)$, e uma cobertura considerada completa, como a do município Araçuaí (92,1\%) (Tabela 1).

Dos 485 NV não informados ao SINASC, quase um quarto $(24,3 \% ; n=118)$ não tiveram a DN localizada em nenhuma fonte da busca ativa. Os municípios de Felício dos Santos e Jordânia foram os que apresentaram maior proporção de DN não localizadas na busca ativa em relação aos NV não informados, correspondendo também aos municípios com maior proporção de subenumeração (Tabela 1).

Analisando-se o número de fontes de busca ativa nas quais os $\mathrm{NV}$ foram encontrados, verificou-se que aproximadamente $30 \%$ dos casos subenumerados foram localizados em apenas uma fonte e aproximadamente $70 \%$ puderam ser encontrados em até três fontes. Considerando os casos que não tiveram DN emitida, a proporção localizada em apenas uma fonte é maior $(63,6 \%)$, sendo que mais de $90 \%$ dos casos puderam ser localizados em até três fontes
(Tabela 2).

Quanto à contribuição das principais fontes de informação pesquisadas, destacaram-se os cartórios de registro civil, que contribuíram com $42,7 \%$ $(\mathrm{n}=207)$ dos NV não informados ao SINASC, seguido das Unidades Básicas de Saúde (40,8\%, $\mathrm{n}=198)$ e dos hospitais $(37,1 \%, \mathrm{n}=180)$. Essas três fontes, quando somadas, foram responsáveis por $60 \%$ dos registros localizados na busca ativa.

Avaliando a frequência de localização dos NV em mais de uma fonte, verificamos que, dos 207 nascidos localizados no cartório, 66 foram localizados exclusivamente nessa fonte, 113 foram encontrados também em Unidades Básicas de Saúde, $100 \mathrm{em}$ hospitais, $34 \mathrm{em}$ arquivos das secretarias municipais de saúde, 27 em igrejas, 23 em programas de saúde da família e 17 em registros de serviços de assistência social (Tabela 3). Quando o nascimento foi localizado em apenas uma fonte, o cartório permaneceu como principal fonte $(n=66)$, seguido da unidade básica de saúde $(\mathrm{n}=26)$ e do hospital ( $\mathrm{n}=24)$.

Dos NV que não tiveram DN emitida, as principais fontes foram os cartórios $(36,4 \%)$, o Programa de Saúde da Família - PSF (29,7\%) e as Unidades Básicas de Saúde (22,0\%). Um achado importante foi que $18,1 \%$ dos NV localizados por busca ativa tinham DN emitida e localizada em arquivos das Secretarias Municipais de Saúde (SMS).

Tabela 1

Número e proporção de nascidos vivos informados ao SINASC e de nascidos vivos localizados por busca ativa, segundo emissão de Declaração de Nascido Vivo (DN). Vale do Jequitinhonha, Minas Gerais, Brasil, 2008.

\begin{tabular}{|c|c|c|c|c|c|c|c|}
\hline \multirow[t]{2}{*}{ Município } & \multirow{2}{*}{$\begin{array}{c}\text { Nascidos vivos } \\
\text { informados ao } \\
\text { SINASC }\end{array}$} & \multicolumn{4}{|c|}{ Nascidos vivos localizados pela busca ativa } & \multirow{2}{*}{$\begin{array}{c}\text { Proporção de } \\
\text { subenumeração }\end{array}$} & \multirow[t]{2}{*}{ Coberturas(\%) } \\
\hline & & DN emitida & $\% a$ & DN não emitida & $\% a$ & & \\
\hline Araçuaí & 582 & 44 & 7,0 & 6 & 0,9 & 7,9 & 92,1 \\
\hline Felício dos Santos & 70 & 32 & 26,7 & 18 & 15,0 & 41,7 & 58,3 \\
\hline Joaíma & 161 & 111 & 40,8 & - & - & 40,8 & 59,2 \\
\hline Jordânia & 72 & 109 & 45,0 & 61 & 25,2 & 70,2 & 29,8 \\
\hline Novo Cruzeiro & 610 & 71 & 9,9 & 33 & 4,6 & 14,6 & 85,4 \\
\hline Total & 1495 & 367 & 18,5 & 118 & 6,0 & 24,5 & 75,5 \\
\hline
\end{tabular}

a O cálculo do percentual é relativo à soma dos valores dos nascidos vivos não informados e dos nascidos vivos informados;

b Para o cálculo de cobertura, no numerador foi utilizado o número de nascidos vivos informados ao SINASC e, no denominador, foram considerados os nascidos vivos informados ao SINASC acrescidos dos nascidos localizados por busca ativa. 
Distribuição de nascidos vivos localizados por busca ativa, segundo número de fontes de busca ativa onde foram encontrados e emissão de Declaração de Nascido Vivo (DN). Vale do Jequitinhonha, Minas Gerais, Brasil, 2008.

\begin{tabular}{|c|c|c|c|c|}
\hline \multirow[t]{3}{*}{ Número de fontes } & \multicolumn{4}{|c|}{ Nascidos vivos localizados pela busca ativa } \\
\hline & \multicolumn{2}{|c|}{ Total } & \multicolumn{2}{|c|}{ NV cuja DN não foi emitida } \\
\hline & $\mathrm{n}$ & $\%$ & $\mathrm{n}$ & $\%$ \\
\hline Uma & 147 & 30,3 & 75 & 63,6 \\
\hline Duas & 112 & 23,1 & 21 & 17,8 \\
\hline Três & 90 & 18,6 & 13 & 11,0 \\
\hline Quatro & 73 & 15,1 & 9 & 7,6 \\
\hline Cinco ou mais & 63 & 13,0 & - & - \\
\hline Total & 485 & 100,0 & 118 & 100,0 \\
\hline
\end{tabular}

$\mathrm{NV}=$ Nascidos vivos

Tabela 3

Distribuição de nascidos vivos localizados por busca ativa, segundo fontes de busca ativa onde foram encontrados e emissão de Declaração de Nascido Vivo (DN). Vale do Jequitinhonha, Minas Gerais, Brasil, 2008.

\begin{tabular}{|c|c|c|c|c|c|c|c|}
\hline Fontes de informação & Cartório & UBS & Hospital & SMS & PSF & Assistência social & Igreja \\
\hline \multicolumn{8}{|c|}{ NV localizados apenas em uma fonte } \\
\hline NV com emissão de DN & 33 & 4 & 19 & 1 & - & 7 & 8 \\
\hline NV sem emissão de DN & 33 & 22 & 5 & - & 6 & 7 & 2 \\
\hline \multicolumn{8}{|c|}{ NV encontrados em uma ou mais fontes } \\
\hline Cartório & 207 & 113 & 100 & 34 & 23 & 17 & 27 \\
\hline UBS & - & 198 & 123 & 71 & 61 & 30 & 39 \\
\hline Hospital & - & - & 180 & 77 & 42 & 47 & 51 \\
\hline SMS & - & - & - & 88 & 40 & 42 & 25 \\
\hline PSF & - & - & - & - & 76 & 31 & 26 \\
\hline Assistência Social & - & - & - & - & - & 69 & 24 \\
\hline Igreja & - & - & - & - & - & - & 69 \\
\hline
\end{tabular}

Os dados da linha e coluna não correspondem ao total de nascidos vivos identificados na busca ativa porque o mesmo nascido vivo pode ser encontrado em mais de uma fonte.

NV= Nascidos vivos; USB= Unidades Básicas de Saúde; SMS= Secretarias Municipais de Saúde; PSF= Programa de Saúde da Família.

\section{Discussão}

Os resultados deste estudo indicam uma cobertura precária do SINASC, muito aquém da cobertura de 90\% preconizada pelo Ministério da Saúde para uso do dado no cálculo direto de indicadores de saúde. 19 Coberturas menores que $40 \%$ encontradas em três dos cinco municípios pesquisados, são bem inferiores às apontadas em estudos mais recentes sobre o tema, mesmo em regiões de porte populacional semelhante. $8,15,19$ Apesar do país nos últimos anos ter apresentado importantes avanços na cobertura e completitude dos dados, ${ }^{8}$ a subenumeração de eventos vitais ainda é uma realidade, principalmente em regiões menos desenvolvidas. $7,8,10$

A proporção de partos hospitalares nesses municípios, no ano de 2008 , era de $96 \%$, muito semelhante à do país como um todo. Apesar disso, verificou-se elevado número de nascidos vivos cuja DN não foi emitida por nenhum serviço de saúde. Sabe-se que, mesmo em caso de partos domiciliares, a declaração de nascido vivo pode ser preenchida pela unidade de saúde que toma conhecimento do caso. ${ }^{6}$ Contudo, observou-se ainda grande proporção 
desses registros encontrada em estabelecimentos de saúde. Esse dado sugere a existência de hiatos entre a assistência e a vigilância em saúde, bem como indica que o município não realiza busca ativa de nascimentos como preconizado pelo Ministério da Saúde, 20 merecendo atenção dos gestores locais. Essa subenumeração gera obstáculos tanto ao uso de indicadores demográficos e de saúde, como a intervenções locais oportunas no âmbito da saúde materna e infantil, opondo-se ao objetivo da implantação desse sistema que é descentralizado e rico em informações.

É também relevante a proporção de nascidos vivos que tiveram as DN emitidas, mas que não foram inseridas no SINASC. O achado de $18 \%$ dos nascidos vivos subenumerados terem DN emitida e arquivada nas SMS sugere grande deficiência na execução do processo de busca, notificação e digitação de dados no nível local e pode indicar deficiência de recursos humanos capacitados para a realização das atividades. Pressupõe ainda falta de integração entre os níveis de gestão e desconhecimento do nível estadual sobre a fragilidade municipal no cumprimento do fluxo de transmissão das informações, evidenciando a necessidade de mudanças no processo de trabalho no âmbito local e maior acompanhamento pelas demais esferas de gestão. Estudos qualitativos como o de Guimarães et al.21 e de Campos et al.22 sobre o contexto organizacional dos sistemas de informações SIM e SINASC em municípios brasileiros, descreveram as dificuldades em relação à ausência de profissionais capacitados para a operacionalização dos sistemas de informação, bem como a alta rotatividade de pessoal nas atividades de vigilância. Os estudos também mostraram limitações ainda quanto à infraestrutura e suporte técnico, como a dificuldade com carros para a atividade de busca ativa, problemas de instalação de programas para digitação de dados no sistema e ausência de internet. Problemas semelhantes foram observados durante a busca ativa nos cinco municípios pesquisados e relatados nos diários de campo.

Em relação à coleta de informações, os cartórios e os serviços de saúde representaram mais da metade dos registros não informados ao SINASC. Esse achado vai ao encontro com o que é apontado na literatura para a busca ativa de óbitos. $8,19,23,24$ São nascimentos que foram registrados em cartório ou tiveram assistência em serviços de saúde, que por sua vez não preencheram a DN. Semelhante ao fluxo de distribuição de Declarações de Óbitos (DO), as DN são distribuídas gratuitamente aos estabelecimentos de saúde e cartórios pelas Secretarias Municipais de Saúde.25 Portanto, mediante a incerteza do preenchimento desses documentos, a busca nos livros de registros dos cartórios e em outros meios disponíveis nas unidades de saúde é recomendada.

O Programa de Saúde da Família e os serviços municipais de assistência social, ainda que em menor proporção, mostraram-se fontes relevantes para a busca de nascidos vivos nos municípios estudados. Os agentes comunitários de saúde, devido à proximidade e constante contato com a comunidade, foram de grande importância na localização dos casos e na confirmação domiciliar. O Ministério da Saúde sugere que os dados coletados mensalmente pelos profissionais da Estratégia de Saúde da Família sejam utilizados para o aperfeiçoamento do SINASC e melhoria da cobertura nos municípios onde o Sistema de Informação da Atenção Básica foi implantado. 25

Uma limitação do estudo foi que, ainda que distritos distantes das sedes dos municípios tenham sido visitados durante a coleta de dados, em algumas localidades houve grande dificuldade de acesso e transporte, especialmente pela pesquisa se dar em época chuvosa, em sua maioria zonas rurais, onde se espera uma importante subenumeração dos nascimentos, o que pode ter acarretado na não captação de algum NV de famílias residentes nessas áreas. Tal dificuldade sazonal também é experimentada pela população e pelos servidores municipais encarregados da notificação dos eventos vitais. Porém, isso não justifica a não informação, pois a digitação dos dados no sistema computacional pode ser realizada a qualquer tempo. O pequeno número de eventos nos pequenos municípios não deve trazer dificuldades mesmo quanto à questão de deficiência de recursos humanos na área de vigilância. A busca ativa de nascimentos pode ser incorporada à rotina da própria vigilância epidemiológica de agravos ou à rotina de outras ações em saúde pública.

Faz-se importante ressaltar que, como a pesquisa é referente a eventos ocorridos em 2008, a situação de saúde, organização dos serviços e o grau de consolidação do SINASC nesses municípios podem ter sofrido alterações. A série temporal de 2008 a 2013, disponibilizada na página do DATASUS (dados não mostrados), aponta que ainda há grandes divergências entre o volume de NV informados ao SINASC nacional e o total de NV após busca ativa. É possível observar também considerável variação de registros entre os anos na maioria dos municípios. Esses dados podem indicar a persistência de possíveis irregularidades nesse sistema, a não sistematização da prática de busca ativa ou mudanças no comportamento demográfico desses 
municípios.

Conclui-se que o produto da busca ativa de nascidos vivos demonstra a relevância de uma ação mais consistente no âmbito da qualidade dos registros vitais em municípios de pequeno e médio porte no país, uma vez que gera mudanças expressivas no perfil demográfico dessas regiões com precariedade na informação. A qualificação do SINASC permite o uso dos dados desse sistema para as diversas ações de vigilância em saúde, de assistência à saúde materno-infantil e avaliação

\section{Referências}

1. Laurenti R, Jorge MHPM, Lebrão ML, Gotlieb SLD, Almeida, MF. Editorial Especial - Estatísticas Vitais: contando os nascimentos e as mortes. Rev Bras Epidemiol. 2005; 8 (2): 108-10.

2. RIPSA (Rede Interagencial de Informação para a Saúde). Indicadores básicos para a saúde no Brasil: conceitos e aplicações / Rede Interagencial de Informação para a Saúde Ripsa. 2 ed. Brasília, DF: Organização Pan-Americana da Saúde; 2008. 349 p.

3. Romero DE, Cunha CB. Avaliação da qualidade das variáveis epidemiológicas e demográficas do Sistema de Informações sobre Nascidos Vivos, 2002. Cad Saúde Pública. 2007; 23 (3): 701-14.

4. Szwarcwald CL. Strategies for improving the monitoring of vital events in Brazil. Int J Epidemiol. 2008; 37 (4): 73844

5. IBGE (Instituto Brasileiro de Geografia e Estatística). Indicadores sociodemográficos e de saúde no Brasil 2009. Série Estudos e Pesquisas - Informação Demográfica e Socioeconômica; 2009. 152.

6. Brasil. Ministério da Saúde. Manual de procedimentos do sistema de informações sobre nascidos vivos. Brasília, DF Ministério da Saúde, Fundação Nacional de Saúde; 2001.

7. Mello Jorge MHP, Laurenti R, Gotlieb SLD. Análise da qualidade das estatísticas vitais brasileiras: a experiência de implantação do SIM e do SINASC. Ciênc Saúde Coletiva. 2007; 12 (3): 643-54.

8. Szwarcwald CL, Morais Neto OL, Frias PG, Souza Junior PRB, Escalante JJC, Lima RB, Viola RC. Busca ativa de óbitos e nascimentos no Nordeste e na Amazônia Legal: estimação das coberturas do SIM e do SINASC nos municípios brasileiros. In: Ministério da Saúde, Departamento de Análise de Situação de Saúde, Secretaria de Vigilância em Saúde, (org.). Saúde Brasil, 2010: uma análise da situação de saúde e de evidências selecionadas de impacto de ações de vigilância em saúde. Brasília: Ministério da Saúde; 2011. p. 79-98.

9. Andrade CLT, Szwarcwald CL. Desigualdades sócio-espaciais da adequação das informações de nascimentos e óbitos do Ministério da Saúde, Brasil, 2000-2002. Cad Saúde Pública. 2007; 23: 1207-16.

10. Rede Interagencial de Informação para a Saúde (RIPSA). Indicadores e dados básicos para a saúde (IDB) 2009. [acesso em 20 jul 2013]. Disponível em: http://tabnet.datasus.gov.br/cgi/idb2009/matriz.htm. dessas ações, contribuindo com informações oportunas e de qualidade para as políticas de saúde tanto no nível local, quanto em níveis estaduais e nacional. Sugere-se a busca ativa como rotina pelos municípios, ainda que concentradas nas fontes principais, como cartórios, unidades de saúde e hospitais. Consideramos ser esse um passo fundamental na elaboração de estratégias adequadas aos serviços de saúde para a disponibilização de informações de maior qualidade nos diversos níveis de gestão.

11. Romero DE. Vantagens e limitações do método demográfico indireto e dos dados da PNAD's 98 para estimativa da mortalidade infantil. In: Encontro da Associação Brasileira de Estudos Populacionais; 2002; Ouro Preto, Brasil. Anais. Ouro Preto, 2002. [on line]. [acesso em 06 ago 2013]. Disponível em: http://www.abep.nepo.unicamp.br/docs/ anais/pdf/2002/gt_sau_st3_romero_texto.pdf.

12. Victora CG, Aquino EM, Leal MC, Monteiro CA, Barros FC, Szwarcwald CL. Maternal and child health in Brazil: progress and challenges. The Lancet, 2011. v. 377, n.9780, p.1863-76.

13. Frias PG, Mullachery PH, Giugliani ERJ. Políticas de Saúde direcionadas às crianças brasileiras: breve histórico com enfoque na oferta de serviços de saúde. In: Ministério da Saúde, Departamento de Análise de Situação de Saúde, Secretaria de Vigilância em Saúde, (org.). Saúde Brasil, 2008. 20 anos de Sistema Único de Saúde (SUS) no Brasil. Brasília, DF: Ministério da Saúde; 2009. p.85-110.

14. Frias PG, Pereira MH, Andrade CLT, Szwarcwald CL. Sistema de Informações sobre Mortalidade: estudo de caso em municípios com precariedade dos dados. Cad Saúde Pública. 2008; 24: 2257-66.

15. Drumond, EF, Machado CJ, França EB. Subnotificação de nascidos vivos: procedimentos de mensuração a partir do Sistema de Informação Hospitalar. Rev Saúde Pública. 2008; 42 (1): 55-63.

16. Schimid B, Silva NN. Estimação de sub-registro de nascidos vivos pelo método de captura e recaptura, Sergipe. Rev Saúde Pública. 2011; 45(6): 1088-98.

17. Nascimento EC. Vale do Jequitinhonha: entre a carência social e a riqueza cultural. Rev Artes Humanidades. 2009; 4: 1-15.

18. IBGE (Instituto Brasileiro de Geografia e Estatística). Censo Demográfico 2010. [acesso em 20 mai 2015]. Disponível em: www.censo2010.ibge.gov.br.

19. Frias PG, Pereira PM, Vidal AS, De Lira PIC. Avaliação da cobertura do Sistema de Informações Sobre Nascidos Vivos e a contribuição das fontes potenciais de notificação do nascimento em dois municípios de Pernambuco, Brasil. Epidemiol Serv Saúde. 2007; 16: 93-101.

20. Brasil. Ministério da Saúde. Portaria $n^{\circ} 116$, de 11 de fevereiro de 2009. Regulamenta a coleta de dados, fluxo e periodicidade de envio das informações sobre óbitos e nascidos vivos para os Sistemas de Informações em Saúde 
sob gestão da Secretaria de Vigilância em Saúde. Brasília, DF. [DOU]; 2009.

21. Guimarães EAA, Carmo RF, Loyola Filho AI, Meira AJ, Luz, ZMP. O contexto organizacional do Sistema de Informações sobre Nascidos Vivos segundo profissionais de saúde do nível municipal. Rev Bras Saúde Mater Infant. 2014; 14 (2): 165-72.

22. Campos D, Hadad SC, Abreu DMX, Cherchiglia ML, França EB. Mortality Information System in small municipalities of Minas Gerais state: concepts of health professionals. Ciênc Saúde Coletiva. 2013; 18 (5): 1473-82.

23. Cunha CC, Campos D, França EB. Uso da busca ativa de óbitos na avaliação do Sistema de Informações sobre Mortalidade em Minas Gerais, Brasil. Epidemiol Serv Saúde. 2011; 20: 275-86

Recebido em 14 de julho de 2015

Versão final apresentada em 14 de agosto de 2015

Aprovado em 21 de agosto de 2015
24. Figueiroa BQ, Vanderlei LCM, Frias PG, Carvalho PI, Szwarcwald CL. Análise da cobertura do Sistema de Informações sobre Mortalidade em Olinda, Pernambuco, Brasil. Cad Saúde Pública. 2013; 29: 475-84.

25. Brasil. Ministério da Saúde. Sistemas de Informações sobre Mortalidade (SIM) e Nascidos Vivos (Sinasc) para os profissionais do Programa Saúde da Família / Ministério da Saúde. Brasília, DF; 2004 